\title{
Michel Cuypers, Le Festin. une lecture de la sourate al- Mā'ida
}

Paris, Lethielleux, Rhétorique sémitique, 2007, 453 p. ISBN :

978-2-283-61251-4.

\section{Anne-Sylvie Boisliveau}

\section{(2) OpenEdition}

\section{Journals}

Édition électronique

URL : http://journals.openedition.org/beo/141

DOI : $10.4000 /$ beo. 141

ISBN : 978-2-35159-260-8

ISSN : 2077-4079

\section{Éditeur}

Presses de l'Institut français du Proche-Orient

\section{Édition imprimée}

Date de publication : 1 janvier 2008

Pagination : 205-209

ISBN : 978-2-35159-038-4

ISSN : 0253-1623

Référence électronique

Anne-Sylvie Boisliveau, «Michel Cuypers, Le Festin. une lecture de la sourate al-Mā'ida », Bulletin d'études orientales [En ligne], Tome LVII | Janvier 2008, mis en ligne le 13 novembre 2009, consulté le 21 septembre 2020. URL : http://journals.openedition.org/beo/141 ; DOI : https://doi.org/10.4000/beo 141 
LE FESTIN. UNE LECTURE DE LA

SOURATE AL-MĀ'IDA

\section{Michel CUYPERS}

Paris, Lethielleux, Rhétorique sémitique, 2007, $453 \mathrm{p}$.

ISBN : 978-2-283-61251-4.

Cet ouvrage est un évènement dans le domaine des études textuelles du Coran. L'auteur y fait clairement la démonstration des fruits que peut porter la méthode dite « analyse rhétorique ». Méthode qui, grâce à des rapports de symétrie aux différents niveaux du texte, permet de retrouver la logique de sa construction, logique obéissant aux règles d'une "rhétorique sémitique » que l'on retrouve dans de nombreux textes du Proche-Orient ancien (Bible, textes ougaritiques et peut-être akkadiens, Hadîth). (voir ci-dessous).

Le Festin cependant est loin d'avoir la sécheresse qu'on pourrait attendre de la présentation technique d'une telle méthode. On y découvre avec grand intérêt une véritable exégèse de la sourate 5 , alimentée notamment par une analyse intertextuelle puisant dans la tradition biblique. Et les données, résultant de la méthode rhétorique, sont en permanence confrontées à - et enrichies par - celles de l'exégèse musulmane classique et moderne et des études orientalistes. On y apprend donc beaucoup.
L'analyse a conduit à relever deux sections (A et B) dans la sourate al-Mâ'ida, composée chacune de trois sous-sections, (comprenant chacune une ou deux séquences: (A1-A2), (A3-A4), (A5), et (B1), (B2), (B3)). Chaque séquence est analysée ici dans un chapitre, dont les divisions traitent des passages qui la constituent. L'analyse remonte ensuite les niveaux : l'ensemble de la séquence, la soussection, la section puis toute la sourate, comme un système de poupées russes. A chaque étape, sont présentés le texte du passage, d'éventuelles questions de vocabulaire, la composition retrouvée par l'analyse, des éléments d'interprétation, et enfin le contexte interscripturaire, quand il y a lieu. Puis l'auteur re-dispose le texte étudié en plusieurs tableaux mettant en avant différents types de symétrie à différents niveaux par des procédés typographiques. On aurait aimé disposer de ces tableaux en arabe, puisque c'est bien sûr sur le texte arabe que l'analyse est basée ; cela aurait permis de surcroît de voir rimes et assonances. On peut s'interroger sur certains 
rapports symétriques trouvés par l'auteur, particulièrement lorsque les symétries concernent le sens des mots ou expressions et sont moins faciles à repérer que les symétries phonétiques ou syntaxiques. Aux niveaux inférieurs, un autre chercheur ne trouverait sans doute pas les mêmes symétries que M. Cuypers, ce qui engendrerait un découpage un peu différent (par exemple, une partie pourrait être considérée par l'un comme comprenant un morceau de deux segments puis un morceau de trois segments, et par l'autre comme comprenant un morceau de trois segments puis un morceau de deux) ; tout au moins, l'un verrait des symétries non-découvertes par l'autre, et vice-versa. Mais aux niveaux supérieurs les doutes sont dissipés : la technicité de l'étude et la précision des symétries dévoilées montrent combien ces liens sont réellement présents dans le texte lui-même.

Le dernier chapitre revient sur la démarche de l'auteur. Puis un appendice, p. 399-416, fait le point sur la question de la cohérence du Coran à travers l'histoire de l'exégèse. Suivent dix pages de bibliographie, puis des index (auteurs cités, références coraniques et bibliques, notions développées).

L'ouvrage présente donc une triple analyse pour chaque passage de la sourate: rhétorique sémitique, exégèse tant classique que moderne, et intertextualité biblique. « Nous n'avions, au départ, pas d'autre intention que d'examiner si et comment une sourate longue, comme la sourate al-Mâ'ida, était composée, et quelle interprétation pouvait découler de la composition du texte ainsi établie. Rapidement cependant s'est imposée la nécessité d'y joindre une analyse intertextuelle » (p. 383), indique l'auteur. On peut penser que si celui-ci se trouve amené à compléter l'analyse rhétorique par des éléments d'exégèse classique et moderne, et par des données issues d'autres textes, c'est parce que l'analyse rhétorique seule ne peut porter de fruits. Peut-être, mais l'analyse rhétorique permet un agencement nouveau des données déjà disponibles (celles de l'exégèse classique ainsi que celles apportées par l'intertextualité). Agencement qui est une véritable clef de compréhension du texte !

Par exemple, une des lois de la rhétorique sémitique est qu' « au centre [d'une structure concentrique] il y a souvent un changement dans le déroulement de la pensée et une idée antithétique est introduite. Après quoi, le déroulement premier est repris et poursuivi jusqu'à ce que le système s'achève. ") (p. 22). Le centre marque également une rupture quant au style et à la sémantique. D'où le fait que ce centre n'a souvent été compris par les savants musulmans anciens que grâce à une « circonstance de la révélation » énoncée tardivement, et par les orientalistes que comme un «verset déplacé ». Pourtant la rhétorique sémitique montre que le centre est la clef de compréhension du texte qui l'entoure : on comprend alors par exemple que le critère par lequel Mahomet peut juger les juifs et les chrétiens (thème principal de la séquence A4), c'est la loi du talion (mise au centre de cette séquence).

De plus, un texte bâti selon la rhétorique sémitique met au centre d'une figure concentrique les éléments qu'il veut mettre en évidence. Dans la sourate al-Mā'ida, on trouve dans les centres des éléments qui ont 
valeur universelle, alors que les extrémités traitent d'éléments circonstanciés et particuliers. Ainsi, il conviendrait lors de l'exégèse de ne pas accorder la même importance à tous les versets : par exemple les considérations générales sur la possibilité pour les juifs, chrétiens et sabéens qui pratiques pieusement leur religion d'être sauvés, considérations situées au centre, auraient plus de valeur que les éléments polémiques qui les encadrent.

Par ailleurs, pour M. Cuypers, le fait que la sourate al-Mā'ida, réputée la dernière ou avant dernière sourate révélée, soit composée selon la même rhétorique que les premières sourates mekkoises (qu'il a précédemment analysées) tend à montrer que toutes les sourates sont construites selon la rhétorique sémitique (p.17). Il y décèle même, grâce à l'intertextualité, les structures d'un discours d'adieu. Une question se pose alors : est-ce seulement chaque sourate qui est construite selon cette rhétorique, ou bien également l'ensemble du canon - lequel aurait peut-être alors fait l'objet d'un travail rédactionnel. Ce qui serait suggéré par la reprise, parfois, d'un mot de la fin d'une sourate dans le début de la suivante. Tout cela reste à explorer.

$\mathrm{Au}$ fil de son analyse, M. Cuypers a remarqué la façon spécifique dont le Coran utilise la rhétorique sémitique : par exemple, il constate que le rythme est souvent tertiaire, et qu'il existe une écrasante majorité de constructions concentriques, à tous les niveaux du texte. Il note aussi que «le Coran a une manière le plus souvent très apparente (...) de manier la rhétorique sémitique. Les brusques changements de sujet, (...) les fréquents changements de personnes, (...) sont autant d'indicateurs du découpage du texte. » (p. 385). Il signale enfin que l'utilisation de tournures décisives, sans appel, et souvent en contradiction entre elles, est courante en langage biblique et n'obligerait ni à les prendre à la lettre ni à avoir recours à un système d'abrogation : il suffirait de " "savoir ce que parler veut dire", dans le langage sémitique » (p. 385).

L'étude intertextuelle elle-même se trouve étoffée par l'analyse rhétorique. M. Cuypers note que le Coran lui-même invite à une étude intertextuelle. Aussi y procède-t-il, cherchant à quels textes se réfère le passage coranique étudié. Mais il va plus loin : il montre que la comparaison avec les textes bibliques se fait aussi sur le plan de la structure sémitique de ces textes. Tout se passe comme si, à l'instar de nombre des textes bibliques, le texte coranique « réécrivait » à sa manière tel ou tel passage biblique dont la structure lui est connue, et même, dont lui sont connues des réécritures déjà effectuées dans d'autres passages bibliques. La recherche intertextuelle procède vraiment par tâtonnements, par exemple avec l'épisode de Caïn (p. 166). Les relations intertextuelles sont très complexes, et M. Cuypers a le mérite de les aborder avec sérieux et profondeur. Il va même jusqu'à se risquer à des comparaisons, comme par exemple celle du petit nombre de juifs qui adhèrent à l'islam avec le « petit reste » des élus mentionné dans la Bible (p. 106).

Notons enfin que la démarche de l'auteur n'est pas de transposer par idéologie des éléments d'exégèse biblique à l'exégèse coranique, mais bien d'utiliser des outils qui 
se montrent pertinents tant pour la Bible que pour le Coran : la rhétorique sémitique et une étude intertextuelle respectueuse et sensée.

En conclusion, nous pourrions reprendre les mots de M. Amir Moezzi, dans la préface de l'ouvrage : "L'objectif de Michel Cuypers dans Le Festin était de savoir si l'analyse rhétorique qu'il applique depuis longtemps aux anciennes et brèves sourates mecquoises était tout aussi pertinente pour une sourate médinoise aussi longue et tardive que la cinquième. A mon sens, il y réussit de manière lumineuse et parfaitement maîtrisée. » (p. III)

Un projet de traduction anglaise serait en cours. Une traduction arabe serait également fort souhaitable. Ceci, afin qu'à la fois des spécialistes en étude textuelle du Coran et des exégètes musulmans puissent utiliser à leur tour la méthode présentée par cet ouvrage : elle constitue un véritable pas en avant pour les études religieuses comme pour la religion.

Anne-Sylvie Boisliveau, IFPO Damas.

\section{La rhétorique sémitique}

La méthode de rhétorique dite «sémitique » a été d'abord utilisée dans les études bibliques, et théorisée notamment par Roland Meynet ${ }^{1}$, qui avec d'autres l'a aussi appliquée à la Sunna ${ }^{2}$; puis M. Cuypers ${ }^{3}$ et ses disciples, dont R. Benzine, l'ont appliquée au texte coranique. Elle tente de répondre à la question : « les différents fragments qui composent [le texte] sont-ils agencés entre eux selon une certaine logique qui confère au texte cohérence et unité ? ${ }^{4}$ » et y répond par l'affirmative, en retrouvant la structure « sémitique » du texte. Ceci, grâce à des outils (rapports de symétries, que ce soit au plan lexical, morphologique, syntaxique, rythmique ou encore au plan du discours) et des lois rhétoriques propres aux

1. MEYNET, R., L'analyse rhétorique. Une nouvelle méthode pour comprendre la Bible. Textes fondateurs et exposé systématique, Paris, Cerf, 1989, 347 p.

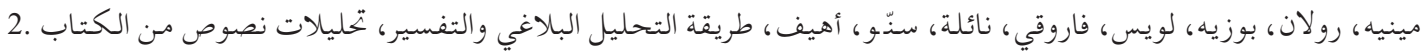
(éd. française : MEYNET, R., POUZET, L., FAROUKI, N., SINNO, A., Rhétorique sémitique. Textes de la Bible et de la Tradition musulmane, Paris, Le Cerf, 1998, 347 p.)

3. M. Cuypers a déjà procédé à des études de sourates courtes, dans les Annales islamologiques de l'IFAO (33, $34,35,37)$ et dans $\operatorname{MIDEO}(22,23,25-26)$, et également dans « Une analyse rhétorique du début et de la fin du Coran », in Al-Kitab. La sacralité du texte dans le monde de l'Islam, Bruxelles-Louvain-La-Neuve-Leuven, de SMET, D., e.a. (éds.), 2004, p. 233-272.

4. Le Festin, p. 13. 
textes du Moyen- Orient ancien, bien différents des outils et lois de la rhétorique grecque. On procède alors au découpage du texte en remontant depuis les niveaux inférieurs, et on établit différents tableaux pour une même portion de texte, afin de montrer les différentes symétries. C'est à cause de cette complexité que la structure du texte n'apparaît pas aux yeux du lecteur, surtout s'il est habitué à une lecture linéaire. «Partir d'une vision générale, c'est à coup sûr risquer un choix arbitraire entre plusieurs structures possibles. Il faut donc impérativement passer par l'austère et laborieux travail de remontée des structures les plus petites vers les plus grandes, en distinguant clairement les divers niveaux textuels, pour s'assurer de l'exactitude du découpage du texte en ses différentes unités, et des relations que ces unités textuelles entretiennent entre elles. ${ }^{5}$ Ainsi, la méthode contient en elle-même bien plus d'objectivité qu'un découpage thématique.

Donnons pour exemple la structure de la sourate al-Fātiḥa, telle qu'analysée par M. Cuypers dans un précédent article ${ }^{6}$. Les rapports entre éléments y sont mis en valeur :

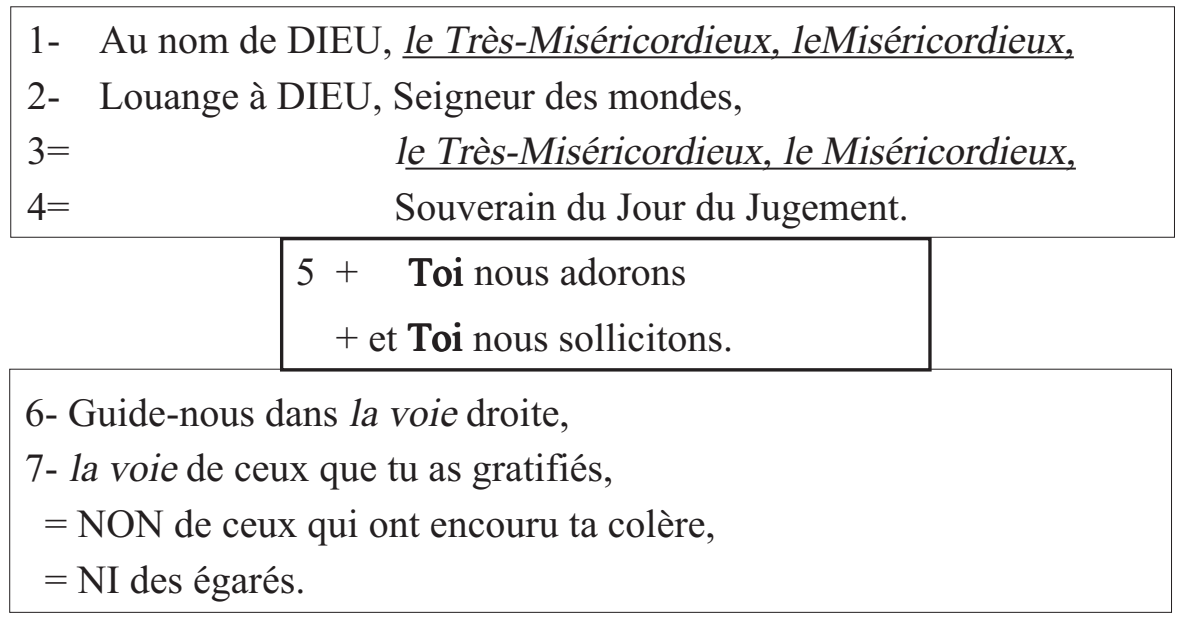

L'intérêt de cette méthode réside dans la réfutation de l'accusation de désordre souvent adressée au texte coranique. Elle permet également de voir l'unité d'un passage, de voir ce qui est « à lire ensemble », ce qui est mis en avant, ce qui est relatif, et même, peut-être, de voir ce qui serait placé dans la structure uniquement par souci de la respecter. Et surtout, elle féconde d'une façon totalement nouvelle les apports des autres types d'analyse.

5. Le Festin, p. 412-413.

6. CUYPERS, M. , « Structures rhétoriques dans le Coran. Une analyse structurelle de la sourate « Joseph » et de quelques sourates brèves », MIDEO, 22 (1994), p. 117. 